\title{
Comparative Study of Data Sharing Based on Network Collaborative Design
}

\author{
Dongfang $\mathrm{Hu}^{1, \mathrm{a}^{*}}$, Minlu $\mathrm{He}^{1, \mathrm{~b}}$ and and Yan $\mathrm{Zhao}^{2, \mathrm{c}}$ \\ ${ }^{1}$ School of Mechatronics Engineering, Henan University of Science and Technology, Luoyang \\ 471003, China \\ ${ }^{2}$ LandGlass Technology Co., Ltd, Luoyang 471003, China \\ ahdf@haust.edu.cn, bhmljdi@163.com, chlxc-2009@163.com
}

Keywords: Data conversion; STEP; XML; Element mapping; Integrated package

\begin{abstract}
The technology of collaborative design based on network has fully strengthened the cooperation ability among enterprises. However, the problem of heterogeneous data sharing among Different design software has been a bottleneck in the development of collaborative design. STEP/XML element mapping method has achieved the conversion between two kinds of data through the establishment of mapping relationship between STEP and XML data language. Because the syntax of the two languages is not the same, it is difficult to ensure the implementation of the mapping. It is likely to cause some data loss in the conversion process. STEP standard update fast and the system is huge, which is hard to achieve comprehensive conversion. Based on the research, the integrated packaging method has been put up in this paper, which is based on CDATA STEP to encapsulate the XML data in XML. Through the comparative study, the integrated packaging method can achieve the integrity of the product model data conversion, which is conducive to the realization of network collaborative design.
\end{abstract}

\section{Introduction}

The integration of network technology and manufacturing industry has made the traditional manufacturing industry develop rapidly, and it is also affecting the competition pattern of the world manufacturing industry. The development of computer technology and communication technology, especially the rapid development and popularization of the Internet in recent years, provides a technical guarantee for online collaborative design. Collaborative design platform will provide professional services to the enterprise grid, and make full use of the technical advantages of the team members to meet the high-end customer personalized needs. Collaborative design platform can effectively use the distributed knowledge resources, and improve the rapid response ability of the enterprise to the market, which is beneficial to the strength of the cooperative members, and improve the agility of enterprises. Because of the habit of collaboration members, the use of the CAD version is not the same. Therefore, the sharing of heterogeneous data has always been a bottleneck in the development of collaborative design. The paper proposed integrated packaging method based on the study of STEP / XML element mapping method. The method is easy to implement, and ensures the integrity of data in the process of conversion, and more effectively to achieve the data sharing among cooperative members.

\section{Selection of XML Expression for EXPRESS Driving Data}

The Application of STEP in Modern Manufacturing Industry. STEP, refers to the standard for the exchange of product model data, is a comprehensive ISO standard that describes how to express and exchange digital product information (ISO 10303), and evolved from previous standards such as VDAFS, IGES, SET, CAD*I, and PDDI ${ }^{[1]}$. STEP is built on the basis of EXPRESS language, the language is a data specification language, composed of a language element defined by a non-ambiguous object and a specification for defining an object. EXPRESS language can describe the data structure of the product, and express its constraints. STEP describe the product life cycle of product data information by providing a neutral mechanism that does not depend on the specific 
system, and most CAD software with import and export STEP data interface. Therefore in the modern manufacturing industry, the use of STEP data throughout the product life cycle, including product design, analysis, manufacture, use, maintenance, scrap, etc.. Currently STEP data mainly in the form of neutral exchange files which named as 10303-21 ISO. It has the following deficiencies:

First, the neutral file is not easy to understand. Specifically, the STEP neutral file is the results of the model which subject to the EXPRESS protocol model. Then, it has no longer reflect the EXPRESS product data information on the structure of the relationship. Second, the level of product data structure is not easy to extract. Because the neutral files saved as the form of ASCII code, and the relationship between the physical elements of the hierarchy and the relationship between the more vague, so it is not easy to read from the file. Third, it is not conducive to network transmission and release. STEP data exist some obstacles in the realization of network sharing, and cannot be published and displayed on the web browser. Therefore, a data which is easy to be transmitted and released by the network is needed to replace the STEP data, so as to better transfer the model information of digital products.

XML Describes the Strong Advantages of Product Data Information. XML is a language that uses text markup to describe data, which has the characteristics of agility, openness, multi-platform, cross-language and so on. XML is a universal data transmission format in today's network, which is proposed by the World Wide Web Consortium. It is widely used in a wide variety of network platforms and systems ${ }^{[2]}$. XML has become the most popular network data interchange format, because it has the following advantages:

(1)The XML file is readability, there is general no binary code in the XML document read, contents of the file exists in text format.

(2)The data structure of XML is clear, and its data structure level is clear, which is called "tree structure".

(3)XML can be constrained by strict syntax, document type of definition (DTD) and architecture (Schema) can not only constrain the XML document, but also describe content structure of the document. They contain the definition rules of elements, relationship rules during the elements, workable attributes, entities and symbols etc. ${ }^{[3]}$.

(4)XML can be used for data exchange between different systems. XML documents can be easily transmitted on the network supporting the HTTP protocol. It is a kind of network service which is built on the simple object access protocol (SOAP). SOAP is an application access protocol, which is labeling rules and processing protocols on the basis of XML.

The Feasibility of Conversion between STEP and XML. Through the above description, we can see that XML has a strong advantage in network transmission, it can be used as the carrier of product data network transmission. EXPRESS language is a formal information modeling language. As the core description language of STEP, it is very similar to XML's syntax data structure, and easy to establish the mapping expression of the basic data types. Both the STEP and XML data structures belong to the tree structure, the level correspondence relationship is quite clear. The data content of STEP and XML is separated from its form, content. The structure of the document are constrained through the framework (Schema).

EXPRESS drives data (ISO 10303-28) expression of XML, it specifies the related standards of model data conversion, which provides the theoretical basis for data conversion from STEP to XML. This standard proposes two kinds of transformation method: pre-binding and late binding. pre-binding: XML tag is corresponding to the data type and attribute in the EXPRESS data model. Late binding: The XML tag is directly corresponding to metadata object of EXPRESS (including entitie, attribute, and data type) ${ }^{[4]}$.

Its EXPRESS mode,

ENTITY person_name;

first : STRING;

middle: STRING;

last : STRING;

END_ENTITY;

The instance data of neutral file are as follows:

$\# 1=$ person_name (Herbert, George, Wells); 
The results of the pre-binding map are as follow:

$\langle$ person_name id="\#1">

$<$ first $>$ Herbert $</$ first $>$

$<$ middle $>$ George $<$ /middle $>$

$<$ last $>$ Wells $</$ last $>$

$</$ person_name $>$

The results of the later stage binding map are as follow:

$\langle$ ENTITY id="\#1" name="person_name">

<attribute name ="first">

$<$ STRING_value $>$ Herbert </STRING_value $>$

$</$ attribute $>$

$<$ attribute name $=$ "middle" $>$

$<$ STRING_value $>$ George </STRING_value $>$

$</$ attribute $>$

$<$ attribute name $=$ "last" $>$ $</$ attribute $>$

$\langle$ STRING_value > Wells </STRING_value >

$</$ ENTITY $>$

By comparison of the two examples above, the output document in early stage is relatively simple, but the document type definition tedious, which must design different XML schema for every entity model, the generality is relatively poor. Although the output document lengthy in late binding, the document type definition is relatively simple, and a definition can be used many times. It can meet the requirement of versatility and portability of data exchange system when realize data exchange.

\section{Advantages of STEP/XML Integrated Packaging Method}

Nowadays, STEP/XML data conversion technology has made a breakthrough. Element mapping method has strong theoretical support, and the method is dominant in the field of research, but it also has its own shortcomings. STEP standard system is huge, establish the perfect mapping relationship is more complex. The integrated package method is relatively simple, easy to implement and good independence, which complete the document does not rely on any framework.

STEP/XML Mapping Method. Mapping method complete the EXPRESS model to XML Schema mapping firstly, which mainly according to corresponding relationship of the EXPRESS mode, patterns, entities, attributes data types, entity references and inheritance relations are mapped into XML markup ${ }^{[5]}$. In the paper, STEP Part 203 for EXPRESS mode is established the corresponding XML Schema according to the STEP Part28 rules. The structure principle is shown in Fig. 1:

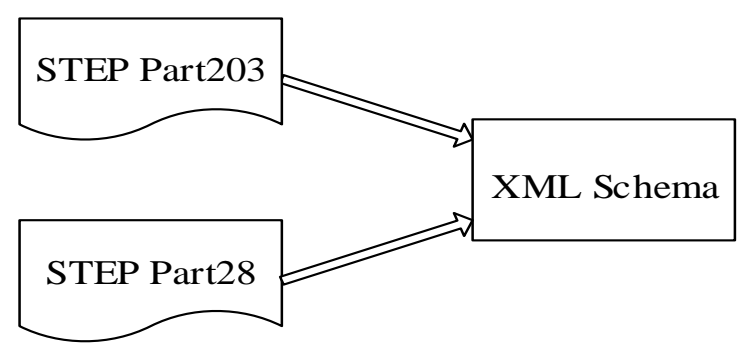

Figure 1. Establish the XML mode structure based on the Part203 STEP and Part28

A simple EXPRESS entity as an example named "person name":

ENTITY person_name;

first: STRING;

middle: STRING;

last: STRING;

END_ENTITY;

Mapped to the corresponding XML Schema form named "person_name.xsd":

$<$ ?xml version=" 1.0 "? >

<xs:schema xmlns:"http://www.w3.org/2001/XMLSchema">

$<$ xs:element name="ENTITY">

$<$ xs:complexType> 


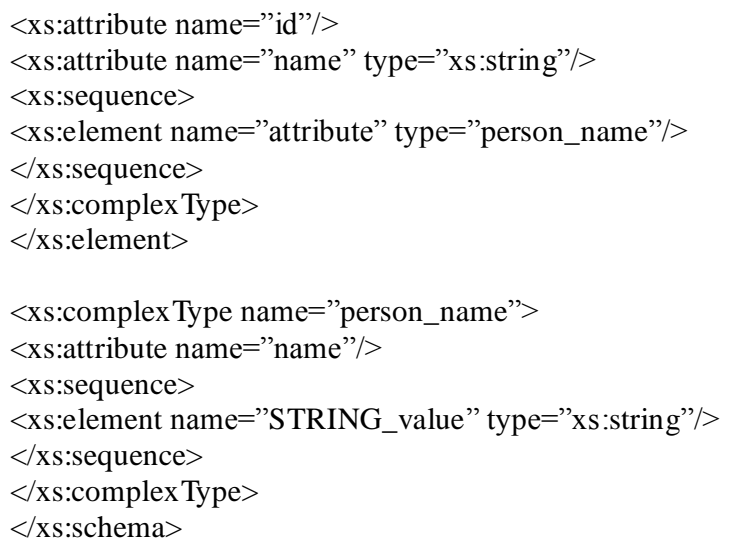

On this basis, with the Schema XML as the constraint rules, mapping conversion is completed from the STEP neutral file to the XML file. The conversion principle is shown in Fig. 2:

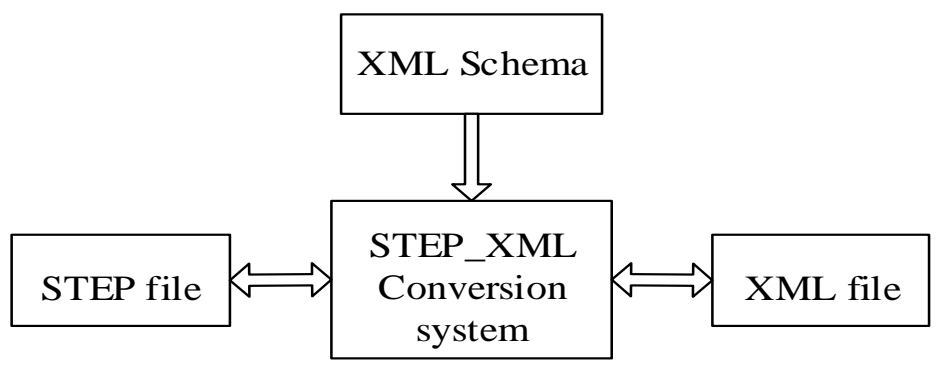

Figure 2 STEP-XML element mapping method data conversion model

STEP_XML Integrated Packaging Method. Completely STEP standard system is extremely large, and multi set standard is still being developed. The DTD or Schema STEP files which are completely corresponding to the full series of STEP of XML have not been worked out. The element mapping method can only convert some of the data in XML ${ }^{[6]}$. Part of the syntax in STEP and XML of these two kinds of data language cannot be achieved one by one. It is likely to cause some data loss in the conversion process. The Integrated package method is to encapsulate the whole STEP data to XML data. The reverse conversion can use DOM to resolve the complete STEP data. This method is easy to implement, and can ensure the integrity of STEP data in the process of conversion. The structure principle of the integrated package method is shown in Fig. 3:

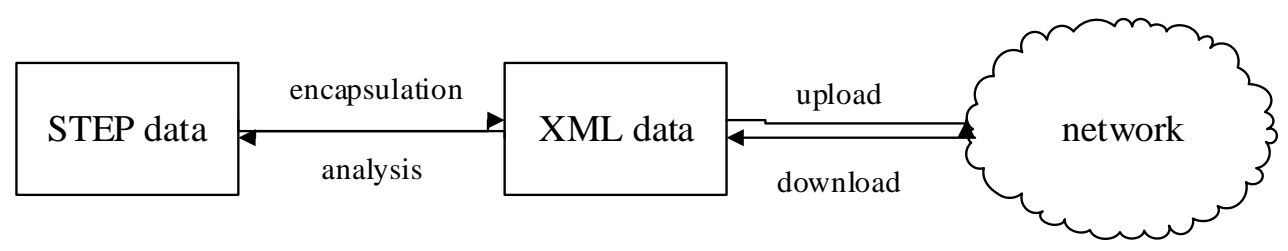

Figure 3. Schematic diagram of the integrated package method

Content between the start tag and the end tag in the element of XML does not appear the markup character and the control character. If there are such characters in the text, this will affect the normal parsing of the XML parser. The character data in XML can solve this problem effectively, and CDATA is the abbreviation of character data (data Character), starting with <! [CDATA[" , and ending with "]]> ". For the XML analyzer, all the text within the CDATA fragment (including the blank and mark characters) should be kept as it is, without any changes ${ }^{[3]}$. In this paper, the overall thought of the package, namely, the step neutral file overall is packaged to the XML character data segment. This scheme ensures the integrity of the data conversion, and conducive to analyze and extract the reduction of DOM XML in the reverse generation of step data.

The document structure after package are as shown as below: 


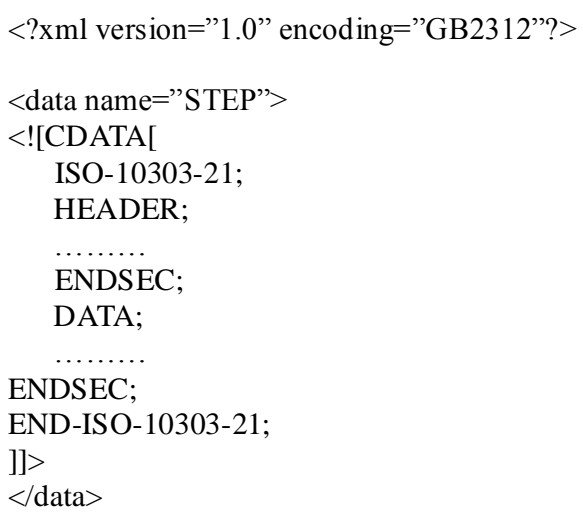

\section{Conclusions}

This paper is based on the network collaborative design platform, and the feasibility of data conversion from STEP to XML is studied. Two methods of element mapping and integrated packaging are introduced for its realization method in detail. In this paper, the Part203 STEP model is mapped by the element mapping method based the theory of STEP Part28, the corresponding XML Schema is produced. On the basis of XML Schema, the STEP neutral file (STEP-21) is converted into the corresponding XML file. The integral packaging method is established based on the theory of XML character data, it packages the STEP neutral file to the XML character data segment. In the reverse process, this paper parses out the corresponding STEP data by the DOM XML parser. Through comparative study, the integral packaging method can ensure the integrity of the data conversion for product model, which is more conducive to the integration of product data model and web visualization, coordination among the members of the coordination design can be achieved better.

\section{Acknowledgment}

The authors gratefully acknowledge the National Nature Science Foundation (Project No. 51575160), the Key Technology R \&D Program of Henan Province (Project No. 13A520232), and the Major and previous pre-research project of Henan University of Science and Technology (Project No. 2011CX016).

\section{References}

[1] Chen Tianfan. Product data exchange technology based on STEP and XML [J]. Machinery design \& Manufacture, 2009, (12): 214-215.

[2] Qiu Xiaoli, Xu Xun, Ni Zhonghua. Digital Product Information Sharing Based on STEP and XML[C]. The 9th International Conference for Young Computer Scientists. 2008

[3] Wang Chengyong, Liu Quankun, Hu Longfei, Hu Chengliang. Research on Data Sharing in Mould Collaborative Design Alliance Based on Web3D/STEP [J]. China Mechanical Engineering, 2006, 17(23): 2477-2481.

[4] Liu Wen, Zeng Qingliang, Fan Wenhui. Research on the WEB-based Product Data Exchange Technology [J]. Machine tool \& Hydraulics, 2008, 36(5): 232-235.

[5] Zhang Yun-sen, LIU Bao-guo, LIU Ke. Research on description and transform technology of product data based on XML and STEP [J]. Manufacture Automation, 2009, 31(3): 36-38, 42.

[6] GB/T 16656.21-2008/ISO 10303-Part 21: Implementation methods-Clear text encoding of the exchange structrue [M]. Standards Press of China, 2008. 